\title{
PENSAR GEOGRÁFICAMENTE EN LA EDUCACIÓN INFAN- TIL: DE LA IMAGINACIÓN A LA CONSTRUCCIÓN SOCIAL DEL ESPACIO CONCEBIDO ${ }^{1}$
}

\author{
Sandra Canet Mahiques²; Antonio José Morales Hernández³; Diego García \\ Monteagudo ${ }^{4}$
}

Recibido: 3/03/2018

Aceptado: 01/07/2018

\section{ReSUMEN:}

La tradición constructivista relaciona la forma de comprender el espacio que tiene el alumnado con su desarrollo físico y psíquico en función de la edad. Pero este planteamiento no explica con suficiente claridad las causas por las que los niños de Educación Infantil son capaces de representar lugares donde nunca han estado o evidenciar relaciones territoriales de cierta complejidad. Por ello hemos abordado un estudio de casos con alumnado de cinco años donde mostramos indicios que permiten evidenciar que el uso de su imaginación en la construcción del espacio concebido puede potenciarse mediante experiencias y recursos didácticos tales como los proyectos de aula y los cuentos.

${ }^{1}$ El presente artículo forma parte del proyecto Interuniversitario "Competencias sociales para una ciudadanía democrática: análisis, desarrollo y evaluación” (COMDEMO - EDU201565621-C3-1-R).

2 Departamento de Didáctica de las Ciencias Experimentales y Sociales (Universitat de València). sancama7@alumni.uv.es

3 Departamento de Didáctica de las Ciencias Experimentales y Sociales (Universitat de València). Antonio.J.Morales@uv.es

${ }^{4}$ Departamento de Didáctica de las Ciencias Experimentales y Sociales (Universitat de València). Diego.Garcia-Monteagudo@uv.es 


\section{Palabras claves:}

Pensar geográficamente, Educación Infantil, imaginación, construcción social, espacio concebido.

\section{Abstract:}

The constructivist tradition relates the way of understanding the space that the students have with their physical and psychic development according to their age. But this approach does not explain with sufficient clarity the reasons why children in childhood education are able to represent places where they have never been or evidence territorial relationships of a certain complexity. For this reason we have addressed a case study with five-year-old students where we show evidence that allows us to show that the use of their imagination in the construction of the conceived space can be enhanced through experiences and didactic resources such as classroom projects and stories.

\section{KEYWORDS:}

Think geographically, childhood education, imagination, social construction, conceived space.

\section{RÉSUMÉ:}

La tradition constructiviste rapporte la manière de comprendre l'espace que les étudiants ont avec leur développement physique et psychique en fonction de l'âge. Mais cette approche n'explique pas avec suffisamment de clarté les raisons pour lesquelles les enfants dans l'éducation infantile sont capables de représenter des lieux où ils n'ont jamais été ou de mettre en évidence des relations territoriales d'une certaine complexité. Pour cette raison, nous avons étudié une étude de cas avec des élèves de cinq ans qui montre que l'utilisation de leur imagination dans la construction de l'espace conçu peut être améliorée par des expériences et des ressources didactiques comme des projets et des histoires en classe.

\section{MoTS CLÉS:}

Penser géographiquement, l'éducation infantile, l'imagination, la construction sociale, l'espace conçu.

\section{JUSTIFICACIÓN Y PLANTEAMIENTO DEL PROBLEMA}

La Psicología evolutiva afirma que existe una relación entre la construcción geométrica-matemática de la concepción del espacio y la edad de las personas. En este sentido cabe citar algunos autores clásicos como Piaget e Inhelder (1947) y otros como 
Hannoun (1977) que categorizan el espacio en; vivido, percibido y concebido. Según esta tradición constructivista en la etapa de Educación Infantil tan sólo se contemplaría, de forma estricta, el espacio vivido. Este planteamiento supone una dificultad para la adquisición de los conocimientos geográficos en la etapa de infantil y no explica con suficiente claridad los motivos por los que los niños/as de Educación Infantil son capaces de representar lugares donde nunca han estado o evidenciar relaciones territoriales de cierta complejidad. Sin embargo, otros autores como Thursthone (1938) o Vigotsky (1962) discrepan que este conocimiento solo se adquiera con la edad, al considerar las experiencias del alumnado como fuente de aprendizaje desde posicionamientos socioconstructivistas. En ambos casos, constructivismo y socioconstructivismo, se aborda el espacio desde el ámbito pedagógico. Por ello también es interesante indagar en el espacio geográfico. Por ello es preciso considerar tanto el espacio absoluto, como el personal o subjetivo (Boira, 2005).

En 1960 Kevin Lynch publica The Image of City y con ello da pie a un nuevo paradigma la Geografía de la Percepción, ofreciendo la posibilidad de que el espacio pueda conceptualizarse como una construcción social. En esta línea trabajarán geógrafos como Boira, Reques y Souto (1994). Precisamente Xosé Manuel Souto (2011) analizará la incidencia de los medios de comunicación en la construcción del conocimiento escolar corroborando con ello la distorsión del concepto de proximidad/ lejanía provocado por la era digital a la que ya aludiera Hernández (2002) y con ello la reconsideración de abordar el conocimiento espacial, no tanto en función de posicionamientos geométricos y/o matemáticos de proximidad, sino más bien de la compresión de la complejidad de las relaciones espaciales tal y como propone Comes (2002).

Peter Gould (2000) manifiesta que nuestra cualidad humana nos habilita para pensar como geógrafos. Cuestión que de alguna manera ya intuye el Convenio Europeo del Paisaje (Consejo de Europa, 2000, p.3) al definir éste como "cualquier parte del territorio tal y como lo percibe la población, cuyo carácter sea el resultado de la acción y la interacción de factores naturales/humanos". En esta definición se relacionan tres conceptos, a saber, paisaje, percepción y territorio, que desvirtúa la concepción estanca del espacio vivido, percibido y concebido transmitida desde la tradición constructivista, para convertirse en algo más dinámico e interactivo. Lana de Souza Cavalcanti (2017) nos ofrecerá tres características clave para trabajar el pensamiento geográfico: lugar, paisaje y territorio. Estas categorías se intentarán evidenciar en las representaciones pictóricas del alumnado de la muestra de esta investigación.

Francesco Tonucci $(1993,1995)$ plantea la necesidad de trabajar el aprendizaje del niños/as a partir de sus propias representaciones mentales porque tal y como manifiesta el autor, si queremos incidir en el ambiente del planeta primero tenemos que hacerlo en el ambiente de los niños/as. Otro pedagogo actual Kieran Egan (2007) nos alienta a utilizar la 
imaginación y la creatividad de los niños/as como una potente herramienta de aprendizaje, vital para la comprensión del espacio en la etapa de Educación Infantil. Todo ello ofrece un abanico de nuevas posibilidades para disponer los recursos educativos que ya conocemos a través de una didáctica más innovadora. Desde este enfoque el curso pasado, en el Máster de Investigación en Didáctica de las Ciencias Sociales (Facultat de Magisteri, València), Estefanía Donet (2016) exponía su Trabajo Final de Máster titulado Pensar històricament en Educació Infantil. Un estudi de cas. Esta cuestión nos alentó a realizar una propuesta, pionera en España, relacionada con la capacidad de desarrollar el pensamiento geográfico en la Educación Infantil, cuyo estudio de casos presentamos como la parte experimental de esta investigación, la cual hemos fundamentando epistemológicamente para este artículo, con la finalidad de mostrar su utilidad didáctica y pedagógica.

¿Es posible pensar geográficamente en la etapa de Educación Infantil? A partir de este interrogante intentamos superar el conocimiento estanco que la tradición constructivista otorga a la Educación Infantil a través de la concepción del espacio vivido. Y a su vez intentamos evidenciar, en este estudio de casos, la aparición de los aspectos relacionados con lugar, paisaje y territorio que Lana de Souza Cavalcanti (2017) propone como propios del pensamiento geográfico. Por todo ello se plantea la siguiente hipótesis: Los alumnos/ as de segundo ciclo de Educación Infantil son capaces de desarrollar el pensamiento geográfico. Para ello el alumnado de cinco años de un Colegio Rural Agrupado (CRA) de la provincia de Valencia ha realizado una serie de representaciones pictóricas del espacio próximo y lejano relacionadas con el "lugar donde viven", "un lugar muy muy lejano" y "ellos/as en un lugar muy muy lejano". Entre los dos dibujos primeros y el último se ha ejecutado una intervención en el aula con la finalidad de contarles un cuento que les pudiera ayudar a realizar la última representación. La interpretación de los dibujos se ha sometido a un Juicio de Expertos con la intención de que pudieran descubrir las claves del pensamiento geográfico en los dibujos y a la vez categorizar los espacios representados. Y en todo el proceso se ha tenido en cuenta lo que los niños/as querían expresar en sus dibujos utilizando para ello el método de triangulación (alumnado/ investigadora/expertos).

En consecuencia, los objetivos de esta investigación son los siguientes:

1. Analizar los dibujos de los espacios próximos y lejanos realizados por alumnos/as de segundo ciclo de Educación Infantil para determinar los lugares representados.

2. Evidenciar las claves del pensamiento geográfico en las representaciones pictóricas.

3. Categorizar los espacios representados según la conceptualización de espacio vivido, percibido y concebido.

4. Innovar en la utilización de los recursos didácticos para la compresión del espacio geográfico. 


\section{MARCO TEÓRICO}

Las respuestas a cualquier pregunta de investigación requieren fundamentar teóricamente el problema y trazar un método o proceso que nos lleve a alcanzar una solución. En este caso, nos preguntamos si es posible aprender el espacio geográfico en el segundo ciclo de Educación Infantil. El interrogante principal que subyace tras esta cuestión es: ¿Qué escuela de pensamiento geográfico nos permite abordar la enseñanza del espacio en Educación Infantil? La respuesta la encontramos en la geografía de la percepción del comportamiento y su relación con la teoría de las representaciones sociales. Desde estos presupuestos epistemológicos y conscientes de los escasos estudios sobre este tema, vamos a sintetizar las aportaciones teóricas y metodológicas tanto clásicas como actuales que han tratado la enseñanza del espacio geográfico en esta etapa educativa.

\subsection{Epistemología sobre el pensamiento geográfico}

Cualquier investigador/a que aborde la concepción del espacio se posiciona de manera más o menos consciente en una escuela de pensamiento geográfico. Desde la institucionalización de la geografía a mediados del siglo XIX, esta ciencia ha contribuido a dotar a la ciudadanía de una cultura territorial acorde con un sentimiento de identidad patrio. Esta finalidad fue desarrollada por la actividad de las Sociedades Geográficas que reforzaron los intereses imperialistas de las naciones europeas y las exploraciones de los territorios coloniales. Bajo esta óptica los programas de enseñanza geográfica en Educación Primaria y Educación Secundaria fueron el otro componente básico para lograr la institucionalización de esta ciencia (Capel, 1981). A partir de este momento, se han desarrollado dos grandes paradigmas científicos separados (positivismo e historicismo), cuyos investigadores/as han abordado el estudio diferencial de la superficie terrestre y la relación entre el ser humano y el medio como premisas esenciales del objeto de estudio geográfico.

Tras este debate epistemológico la concepción espacial que se ha plasmado en el marco escolar español desde la segunda mitad del siglo XX corresponde a la geografía regional posibilista. Estos presupuestos han calado en la Educación Primaria y Secundaria, pero en el caso de la Educación Infantil se hace necesario ampliar otra justificación ya que, entre otras razones, la geografía no es una disciplina independiente y separada de la etapa. Sin embargo, la inclusión de la geografía en esta primera etapa educativa queda

plenamente justificada por su contribución al desarrollo de la personalidad del niño a través de su integración con el medio en el que vive (Tonda, 2009).

Como el conocimiento geográfico ayuda a los niños y niñas a tomar conciencia de las diferencias globales, al mismo tiempo que favorece el aumento de actitudes de integración 
contribuyendo a desarrollar una educación en valores, los contenidos geográficos deben estar presentes desde la infancia. De hecho, Lana de Souza Cavalcanti (2017) remarca que en la contemplación de la espacialidad de la realidad reside el carácter geográfico de esta ciencia bajo la denominación de geografía social. Por ello rescata tres conceptos (lugar, paisaje y territorio) clásicos en la historia de la disciplina geográfica para desarrollar el pensamiento espacial entre el alumnado mediante una enseñanza constructivista que se inicie en la infancia. De esos tres conceptos, "paisaje" permite recrear una imagen de la superficie terrestre a partir de la observación y percepción social. "Lugar" se convierte en el espacio cotidiano y experimentado por el cuerpo, el movimiento y todo aquello que nos envuelve. Este concepto incluye la dialéctica entre la escala local y global que es objeto de debate en el mundo reciente. En cambio, "territorio" expresa las relaciones de poder que se manifiestan en la delimitación y apropiación de áreas geográficas, por lo que su enseñanza en Educación Infantil nos remitiría a un método tradicional asociado a la finalidad inicial de la ciencia geográfica decimonónica.

Al amparo de las tesis socioconstructivistas, la escuela de la percepción y del comportamiento nos permite explicar las vivencias, emociones e imágenes que experimentan los niños y niñas de Educación Infantil. Por ello, estamos convencidos que existe una vinculación necesaria entre la geografía de la percepción y del comportamiento y la teoría de las representaciones sociales (Souto y García Monteagudo, 2016). Esto supone que la generación del conocimiento espacial es responsabilidad del individuo aunque el aprendizaje se genere en el seno de una comunidad social y cultural. Con ello estamos defendiendo una percepción subjetiva del espacio que requiere una enseñanza que tome como punto de partida la diversidad de concepciones previas que pueden explorarse mediante la comprensión de las representaciones sociales.

Desde esa percepción espacial que actúa como un filtro entre las vivencias del individuo y el espacio concebido, el contexto social y cultural condiciona el aprendizaje del espacio. Por tanto, ya no podemos basarnos únicamente en el principio generador de conocimiento espacial a partir del entorno próximo en los primeros años de escolarización. La influencia de fuentes externas (lugares visitados, medios de comunicación, conversaciones de otras personas), a lo que actualmente añadiríamos (cuentos, cine, TICs, redes sociales...) ya fue señalada por Goodey (1973) como agentes que, junto con las experiencias directas (contacto de primera mano con espacios tanto cercanos como lejanos), ayudan a generar una imagen del mundo desde la infancia. Así que la combinación de experiencias ayuda a los niños y niñas a formar una representación social de espacios lejanos a su realidad inmediata. No obstante, esto no significa impugnar el principio metódico clásico de enseñar desde lo cercano y lo conocido, pues coincidimos con Pérez y Souto (1989) que después deben trabajarse espacios más lejanos a la realidad inmediata de los niños y niñas. 
En consecuencia conviene precisar la reinterpretación de conceptos geográficos claves para esta etapa educativa. El pensamiento sincrético impide la distinción clara entre "lo cercano" y "lo lejano", por lo que Nadal (1999) señala que la percepción espacial entre los infantes generaliza los lugares que no están "aquî" tanto con espacios cercanos a su ciudad como con otros que pueden estar a centenares de kilómetros de su hogar. Pero como la formación del pensamiento espacial en Educación Infantil no ha sido un asunto tratado exclusivamente por los geógrafos tenemos que argumentar con la posición de pedagogos y psicólogos. Eso lo vamos a tratar en el siguiente apartado.

\subsection{Construcción del espacio en infantil}

Aunque no podamos asociar un modelo pedagógico a la escuela de la percepción y del comportamiento, Luis y Urteaga (1982) señalan una conjunción entre esta corriente epistemológica y las teorías de Piaget, bajo un paradigma fenomenológico que facilita la comprensión del espacio absoluto y personal o subjetivo (Boira, 2005) resultando una didáctica medioambiental o pedagogía del medio. Actualmente sabemos que dicha escuela geográfica se enmarca en modelos didácticos más amplios que consideran la participación activa del individuo en el proceso de aprendizaje e incorpora técnicas (esquemas mentales, encuestas...) y enfoques interdisciplinares para desarrollar la observación y exploración del entorno con la finalidad que el niño/a pueda conocer, valorar y transformar el medio (Urkidi, 1994) a partir de las representaciones sociales. Pero hasta llegar a esta premisa que considera que las concepciones espaciales son resultado de representaciones mentales de los sujetos, se han sucedido algunas teorías que conviene tener en cuenta a modo de orientación, sobre todo, con la finalidad de realizar propuestas de innovación didácticas.

Los primeros postulados teóricos sobre el espacio cognitivo vinieron de la psicología evolutiva, cuyas investigaciones defienden que el ambiente social en el que se desarrollan las primeras acciones de la infancia es un condicionante al desarrollo de los niños y niñas. En 1947 Piaget junto con Inhelder, publicaron una epistemología sobre la espacialidad desde un enfoque constructivista. La base empírica de esta psicología se sustenta en investigaciones que analizan la evolución ontogenética de las nociones básicas del pensamiento racional y científico (conceptos de espacio, causalidad, seriación...), por la que la actividad cognitiva de los individuos se produce a partir de esquemas de conocimiento que priman el factor genético sobre los elementos culturales. El conocimiento se construye individualmente mediante los mecanismos de asimilación y acomodación que organizan dichos esquemas y dan lugar a estructuras más complejas. Con el proceso evolutivo de los individuos, cada sujeto llegará a construir tres estructuras que se corresponden universalmente con tres 
estadios evolutivos (sensoriomotor, de inteligencia representacional y de operaciones formales) en los que la interacción entre el sujeto y el medio entra en un nuevo nivel de equilibrio al producirse el paso ascendente de un estadio a otro.

En consecuencia, la concepción piagetiana establece que los niños y niñas comenzarían a formar las primeras ideas espaciales en el estadio preoperatorio (2 a 7 años). Estas nociones iniciales tendrían un carácter topológico y se asocian a la proximidad (cerca/ lejos), la separación y continuidad (límite, frontera), ordenación (sucesión lineal) y el carácter cerrado/abierto e interno o externo de los espacios. En esta línea, Piaget hizo hincapié en la experiencia de los niños con el mundo físico (o mundo de los objetos) en la construcción del pensamiento racional, aunque en este primer estadio las propiedades espaciales no están relacionadas ni con el tamaño ni con la forma de los objetos. Este último aspecto se logrará en el estadio de operaciones concretas, una vez que niños y niñas hayan superado su fase de egocentrismo.

Además de la teoría anterior, se dieron lugar otras contemporáneas a Piaget. En 1938, Thursthone dio cuenta de la complejidad del conocimiento espacial puesto que en sus pruebas sobre inteligencia, trató de incluir tanto la capacidad para comprender el espacio que nos rodea con las localizaciones específicas propias, como la capacidad de manipular los objetos en ese espacio y representarnos a nosotros mismos dentro de ese espacio (Rivero y Gil, 2011). De acuerdo a las referencias espaciales corporales (especialmente la lateralidad) se desarrollaron otras investigaciones que vamos a comparar con los presupuestos piagetianos hasta llegar al paradigma socioconstructivista y a las propuestas más recientes sobre la comprensión del espacio en Educación Infantil.

La comprensión del espacio basada en el cuerpo de los niños y niñas fue explicada por Hannoun (1977) a partir de tres fases sucesivas. La primera es la etapa del espacio vivido, en la que la concepción espacial se forma a partir de la experiencia directa, esto es, en base a la acción de su cuerpo y movimiento sobre el espacio. Esta fase se correspondería con el estadio preoperatorio de Piaget y se desarrollaría durante todo el ciclo de Educación Infantil. La segunda etapa es la del espacio percibido en la que se adquiere la noción de espacio geográfico, distinguiéndose distancias y posiciones entre objetos, incluso los puntos cardinales. Ya no es necesario experimentar con el propio cuerpo para representar objetos en tres dimensiones u orientarse en el espacio, que según el modelo de Piaget equivaldría al estadio de operaciones concretas. La tercera fase es la del espacio concebido. A partir de los 11 años, los adolescentes desarrollan una observación objetiva del espacio, analítica y matemática. Es lo que se conoce en el estadio de las operaciones formales de Piaget como espacio euclidiano, lo que permite la realización de mapas que cumplan con los principios geométricos básicos.

Desde el paradigma socioconstructivista, tenemos que mencionar la importancia del proceso de socialización en la comprensión del espacio durante la infancia. Vigotsky 
(1962) enfatiza la acción de la comunidad social en el desarrollo cognitivo de los infantes, minimizándose así la importancia concedida al pensamiento egocéntrico que queda integrado como un elemento más dentro del sistema complejo que constituye el aprendizaje espacial en las primeras edades. Además del entorno social del niño/a, Pilar Comes ha demostrado la existencia de esquemas espaciales previos que se van modificando mediante acciones educativas y la experiencia propia (Rivero y Gil, 2011). De hecho, la existencia de un conocimiento espacial previo al inicio de la educación formal es un aspecto que ya explicaba Comenio en el siglo XVII desde las ciencias de la educación (Mateos, 2001), y recientemente se viene demostrando por la neurociencia.

En la actualidad se asume que el aprendizaje espacial se genera a través de niveles evolutivos progresivos, en los que la mente va asimilando las tres categorías espaciales (espacio vivido, espacio percibido y espacio concebido) mencionadas. En esa estructura tripartita del espacio, los cambios culturales que se suceden en el entorno de los niños y niñas son un factor relevante en la comprensión del espacio. De nuevo, la consideración del espacio percibido como elemento mediador entre el espacio vivido y el espacio concebido, explica que la comprensión espacial se vea mediatizada por fuentes de información (medios de comunicación, internet, redes sociales, lecturas...) que trascienden la escala local de referencia de los propios niños y niñas. Por ello, Pilar Comes (2002, en Rivero y Gil, 2011) ha señalado acertadamente que el concepto de "entorno" ya no pueda referirse exclusivamente a lo más cercano geográficamente. En consecuencia, propone que la construcción de las relaciones espaciales parta de "lo sencillo" a "lo complejo". De nuevo, emerge la construcción de un espacio (o espacios) a partir de la subjetividad de las personas, y especialmente de sus valores e intereses que se filtran a partir del espacio percibido. Rivero y Gil (2011:41) plantean la necesidad de "reflexionar sobre el carácter social del espacio", porque el alumnado de Educación Infantil otorga una cualidad de seguridad al espacio que conoce. Es en este punto en el que la tarea del maestro o maestra de Educación Infantil tiene que ser necesariamente de adecuación de materiales para llegar a conocer esa representación mental del espacio entre su alumnado.

En definitiva, la enseñanza del espacio no puede basarse en enfoques regionalistas sobre el entorno, sino que tiene que trascender desde lo más local hasta alcanzar una visión global que conecte con el mundo real. La potencialidad del entorno en Educación Infantil ya fue defendida por Piñeiro (1983) y ahora nos aproximamos a reconocer las posibilidades didácticas de la inteligencia espacial de Gardner (2005) en esta mista etapa educativa. Por tanto, la didáctica de las ciencias sociales en Educación Infantil $\mathrm{y}$, especialmente desde la geografía, debe permitir el desarrollo de capacidades que ayuden a la integración social y ciudadana de estos niños y niñas para que se conviertan en miembros activos de la sociedad. Para ello vamos a conocer cuáles son sus representaciones sobre el espacio geográfico. 


\subsection{Representaciones escolares}

Tras haber abordado la conceptualización del espacio geográfico desde una doble vertiente (académica y escolar), vamos a centrarnos en las representaciones sociales y pictóricas con las que hemos desarrollado esta investigación. Para ello comenzaremos definiendo las representaciones sociales y las potencialidades de las técnicas que han permitido llevar a cabo las investigaciones desarrolladas bajo este enfoque.

Por representaciones sociales mencionamos la teoría creada por Serge Moscovici en 1978 para dar cuenta sobre el tipo de producción mental y simbólica presente en el conocimiento de sentido común. Los sujetos construyen las representaciones sociales de los objetos en un determinado contexto social a partir de las informaciones (creencias, valores, tradiciones...) que comparten con un grupo social de referencia. Para este autor, las representaciones sociales "son una modalidad particular del conocimiento, donde su función es la elaboración de los comportamientos y la comunicación entre individuos. La representación es un corpus organizado de conocimientos y una de las actividades psíquicas gracias a la cual los hombres hacen inteligible la realidad física y social, se integran en un grupo o en una relación cotidiana de intercambios, liberan los poderes de su imaginación" (Moscovici, 1978, p.17-18). Por ello, las representaciones sociales son una guía que ayuda a los individuos a aprender e interpretar el mundo, así como a organizar sus conductas y formas de comunicación (Andrade y Moreira, 2017).

En las características de las representaciones sociales se distingue una organización específica cuyos elementos se rigen por unas normas de funcionamiento. Esto es lo que ha desvelado Abric en su teoría del núcleo central, que complementa los fundamentos científicos de Moscovici. Según Abric (1994), los elementos de una representación social están jerarquizados y se organizan en torno a un núcleo duro, a partir del cual se sitúan los elementos periféricos que especifican el sentido de las características que los individuos comparten acerca de un determinado objeto. En este núcleo duro interviene la dualidad del contexto. Por un lado, existe un contexto discursivo en el que intervienen las relaciones entre los sujetos que interactúan bajo una misma condición temporal; por ejemplo, las relaciones educativas sobre la innovación del espacio geográfico en Educación Infantil: un conocimiento académico y otro escolar para ejercer la función docente. Por otro lado, el contexto social en el que se activan o desactivan las representaciones sociales y se generan contradicciones aparentes entre la lógica cognitiva y social en la que existe una influencia de las emociones que fusiona las etapas del espacio vivido y el espacio percibido en una única categoría. En este contexto encontramos la opinión de maestros y maestras que siguen reproduciendo esquemas propios de la geografía regional en Educación Infantil. 
A partir de estas referencias teóricas pretendemos mostrar las representaciones sociales que tienen los alumnos y alumnas de Educación Infantil sobre el espacio geográfico, tanto cercano como lejano. Esas representaciones se basan en sus experiencias, en el entorno, en las emociones y en la creatividad. Por ello, esas creaciones mentales van a ir ligadas a representaciones pictóricas, pues son el medio donde las reflejarán, tal y como apuntan diversas investigaciones (Barraza, 1999). De hecho, es una técnica que permite conocer la influencia de las creencias, valores y normas en la construcción de la noción de espacio entre niños y niñas de Educación Infantil (Ortiz, Prats y Baylina, 2011). En 1998, Trepat y Comes señalaban que "las representaciones sociales de nuestro alumnado tienen un fuerte carácter visual. La televisión, el cine, el vídeo y más recientemente el ordenador, son canales continuos de imágenes espaciales de máxima eficacia comunicativa por su dinamismo y realismo. Nuestros alumnos, desde bien pequeños, ven paisajes remotos, están en contacto con un inmenso banco de información espacial" (Trepat y Comes, 1998, p.163). A estos elementos añadiríamos los teléfonos móviles, tablets y otros artilugios tecnológicos que los niños y niñas comienzan a usar (aunque sea de manera intuitiva) con anterioridad al ingreso en la etapa de Educación Infantil.

En consecuencia, Lana de Souza Cavalcanti (2017) apuesta por incorporar los lenguajes alternativos (poesía, música, juegos digitales...) para conectar con las comunicaciones contemporáneas y desarrollar el aprendizaje de las categorías de espacio percibido y concebido. Así que la interpretación de las representaciones pictóricas requiere la comprensión de la influencia de los imaginarios de cada niño/a, además de la realidad que intentan plasmar en el papel. Por ello, debemos tener presente que "las capacidades imaginativas de los niños no solo tienen aplicación en los relatos fantásticos, sino que también crecen y se desarrollan cuando se aplican a la historia, las matemáticas y las ciencias" (Egan, 2007, p.32-33). Con estas consideraciones se afirma que las representaciones pictóricas son una herramienta de gran utilidad en el aprendizaje del espacio en la geografía escolar si en el método de trabajo consideramos la composición de los dibujos, la perspectiva visual del sujeto y los elementos morfológicos que aluden al espacio.

\section{METODOLOGÍA}

Esta investigación cualitativa pretende evidenciar las capacidades geográficas del alumnado de Educación Infantil, a través de un estudio de casos. Para ello se han analizado las representaciones pictóricas del alumnado de 5 años de un CRA de la provincia de Valencia mediante un método de Triangulación (Denzin, 1970). Por ello hemos sometido los datos ofrecidos por el alumnado y la investigadora responsable del 
trabajo al análisis externo que nos pudiera ofrecer un Juicio de Expertos. De este modo el perfil de la muestra queda definido por el alumnado participante desde el contexto escolar, así como por los expertos que han analizado los dibujos desde el contexto universitario. Cuestión que puede ser resumida de la siguiente forma:

- Ocho alumnos de segundo ciclo de Educación Infantil (5 años) pertenecientes al "CRA Riu Vernissa" de Almiserà y Llocnou de Sant Jeroni (Valencia).

- Cuatro expertos universitarios en la materia de Geografía (alumnado y docentes) de la Universidad de Valencia y la de Bogotá.

Para abordar la conjetura sobre la posibilidad de que se pudiera desarrollar el pensamiento geográfico en la etapa de Educación Infantil, nos pareció oportuno plantear dos partes en el diseño de la experiencia de esta investigación: la primera se llevó en la escuela y la segunda en un seminario realizado en la Facultat de Magisteri (Universitat de València).

La investigación en el centro educativo se nutre de las representaciones pictóricas del espacio próximo y lejano que realiza el alumnado de Educación Infantil. En el momento de realización de este estudio de casos en el aula se estaba trabajando el proyecto de Egipto. Por ello se propone la experiencia escolar mediante tres fases:

a) Fase previa (I y II),

b) Fase de intervención,

c) Fase de síntesis.

En la fase previa (I) se le solicitó al alumnado de cinco años que representaran el lugar donde viven y seguidamente se les pidió que dibujaran un lugar muy, muy lejano (fase previa II). En sesiones posteriores, la investigadora llevó a cabo la fase de intervención en la cual les narró, al alumnado de infantil, el cuento Fent un tomb pel món, Caterina i l'Ós (Pieper, 2000), donde se relatan las aventuras de dos amigos que viajaban por el mundo. En la fase de síntesis se les solicitaba a los niños/as que se dibujaran a ellos/ as mismos/as en un lugar muy muy lejano. En el transcurso de la realización de los dibujos la investigadora instaba a los niños/as para que le explicaran lo que habían querido representar en los mismos. A continuación se relaciona al alumnado con el dibujo que ha realizado, señalamos con un asterisco los casos en los que el alumnado, por causas de enfermedad, no ha podido realizar todas las representaciones pictóricas: 
- Alumno 1 (dibujos 4, 5 y 6 )

- Alumna 2 (dibujos 7, 8 y 9)

- Alumno 3 (dibujos 10, 11 y 12 )

- Alumno 4 (dibujo 13, 14 y 15)

- Alumno 5 (dibujo 16, 17 y 18)

- Alumno 6 (dibujo 19 y 20 )*

- Alumna 7 (dibujo 21)*

- Alumno 8 (dibujo 22)*

La experiencia universitaria se ejecuta a través de un seminario de dos horas en la Facultat de Magisteri (Universitat de València), donde la interpretación de los dibujos del alumnado de Educación Infantil se sometió a un Juicio de Expertos. En esta sesión los expertos/as, docentes de la Universidad Pedagógica de Bogotá y de la Universitat de València así como el alumnado de $4^{\circ}$ Grado de Educación Infantil que quiso asistir (convocado a través del aula virtual), tenían que cumplimentar dos fichas. En la primera reflejaban la evolución de la comprensión de las relaciones espaciales simples a las complejas, tal y como argumentaba Comes (2002), a través del análisis de la evolución de los lugares representados en los dibujos por el alumnado de Educación Infantil. Mientras que en la segunda ficha debían analizar en los dibujos la posible relación de los conceptos básicos (lugar, paisaje y territorio) que definen el pensamiento geográfico, según Lana de Souza Cavalcanti (2017) con la categorización del espacio vivido, percibido y concebido en atención a las orientaciones de Hannoun (1977). Todo ello nos ha permitido a los autores de este artículo, una maestra de Educación Infantil y dos profesores que imparten docencia en el Grado de Infantil de la Facultat de Magisteri (Universitat de València), demostrar una serie de indicios relacionados con la imaginación infantil y la construcción del espacio geográfico a través de este estudio de casos.

\section{ANÁLISIS Y DISCUSIÓN DE LOS RESULTADOS}

En este apartado centraremos el análisis de los datos en tres cuestiones relacionadas con las representaciones pictóricas del alumnado: la evolución de los lugares representados; la aparición de aspectos básicos que definen el pensamiento geográfico (lugar, paisaje y territorio) y la categorización de los espacios representados (vivido, percibido y concebido). El contraste de la información de la investigadora, que sabía lo que los niños/as querían representar en sus dibujos porque se lo habían dicho, con el análisis de los expertos/as ha permitido una correcta interpretación de los datos. 


\subsection{Evolución de los lugares representados}

La tabla 1 nos permite evidenciar los lugares que el alumnado ha representado en cada uno de los dibujos, donde se constata que diferencian claramente los espacios próximos y los lejanos. Asimismo se aprecia que se ayudan de experiencias externas como puedan ser los viajes, los medios de comunicación, el proyecto de aula y el cuento que hemos utilizado en la intervención para construir las relaciones espaciales entre lo cercano y lo lejano. En el caso de la representación de "el lugar donde viven" todo el alumnado ha dibujado casas para representar su pueblo. Es un espacio conocido en el que no aparecen personas. Por su parte al representar "un lugar muy, muy, pero que muy lejano se han apoyado en el proyecto de aula sobre Egipto (África) en los viajes que han realizado (Granada, montaña, Londres, Marina d'Or) y en los medios de comunicación al representar un partido de fútbol (Roma). En lo que respecta a la representación de "él/ella en un lugar muy, muy, pero que muy lejano" se han ayudado de sus experiencias y viajes (montañas, Inglaterra) pero también del proyecto de aula (Egipto) y del cuento utilizado en la intervención (India y Everest). En estos casos ellos/as se representan junto a sus familias para sentirse seguros. Por tanto, se observa una evolución de la compresión del espacio de lo más simple a lo más complejo (Comes, 2002) a partir de una construcción social del espacio (Rivero y Gil, 2011), en la que los/las docentes podemos contribuir con los recursos didácticos apropiados que les permitan concebir espacios a partir de su imaginación.

\begin{tabular}{|c|c|c|c|c|}
\hline \multirow{2}{*}{$\begin{array}{l}\text { REPRESENTACIÓN } \\
\text { DE: } \\
\text { El lugar dónde vive }\end{array}$} & \multirow{2}{*}{$\begin{array}{c}\mathrm{N}^{\mathbf{0}} \text { DE } \\
\text { ALUMNOS } \\
\text { QUE LA HAN } \\
\text { REALIZADO } \\
5\end{array}$} & \multicolumn{2}{|c|}{$\begin{array}{l}\text { NOMBRE DE LOS } \\
\text { LUGARES Y No DE } \\
\text { VECES QUE SE HAN } \\
\text { REPRESENTADO EN } \\
\text { CADA DIBUJO }\end{array}$} & \multirow{2}{*}{$\begin{array}{c}\text { ALUMNADO } \\
\text { Alumnos } 1,2,3,4 \text { y } 5\end{array}$} \\
\hline & & LLOCNOU & 5 & \\
\hline \multirow{6}{*}{$\begin{array}{l}\text { Un lugar muy, muy, } \\
\text { pero que muy lejano }\end{array}$} & \multirow{6}{*}{6} & ÁFRICA & 1 & Alumno 1 \\
\hline & & GRANADA & 1 & Alumna 2 \\
\hline & & MONTAÑA & 1 & Alumno 3 \\
\hline & & LONDRES & 1 & Alumno 4 \\
\hline & & ROMA & 1 & Alumno 5 \\
\hline & & MARINA D'OR & 1 & Alumno 6 \\
\hline \multirow{5}{*}{$\begin{array}{l}\text { Él/ella en un lugar } \\
\text { muy, muy, pero que muy } \\
\text { lejano }\end{array}$} & \multirow{5}{*}{8} & INDIA & 2 & Alumnos 1 y 4 \\
\hline & & MONTAÑAS & 1 & Alumna 2 \\
\hline & & INGLATERRA & 1 & Alumno 3 \\
\hline & & EGIPTO & 2 & Alumno 5 y alumna 7 \\
\hline & & EVEREST & 2 & Alumnos 6 y 8 \\
\hline
\end{tabular}

TABLA N $^{0} 1$. Lugares representados por el alumnado de Educación Infantil.

Fuente: elaboración propia 
Para reflejar la construcción de estas relaciones de complejidad entre lo próximo y lo lejano analizaremos de forma más exhaustiva cuatro figuras del estudio que nos han llamado particularmente la atención.

De este modo y en primer lugar, observaremos la Figura 1, en la que el alumno 4 ha representado el lugar dónde vive. Se trata de la representación de su pueblo. En la misma aparece su casa y la de su vecino en la plaza del pueblo dónde reside, obviando los seres vivos, la naturaleza o las personas. Además, se pueden destacar la gran cantidad de nubes dibujadas, así como los tres soles y sus tonalidades, ya que de izquierda a derecha están pintados de más claros a más oscuros, lo que nos hace pensar que se pretende diferenciar el paso del tiempo, o mejor dicho, el paso del día a la noche, por las posiciones y colores, desde que amanece hasta que anochece. Por tanto, vemos que el alumno percibe este paso del tiempo y sabe plasmarlo, además de demostrar una percepción subjetiva del espacio vivido, que evidencia una cierta organización territorial del pueblo, al dibujar las casas y la plaza conformando su barrio.

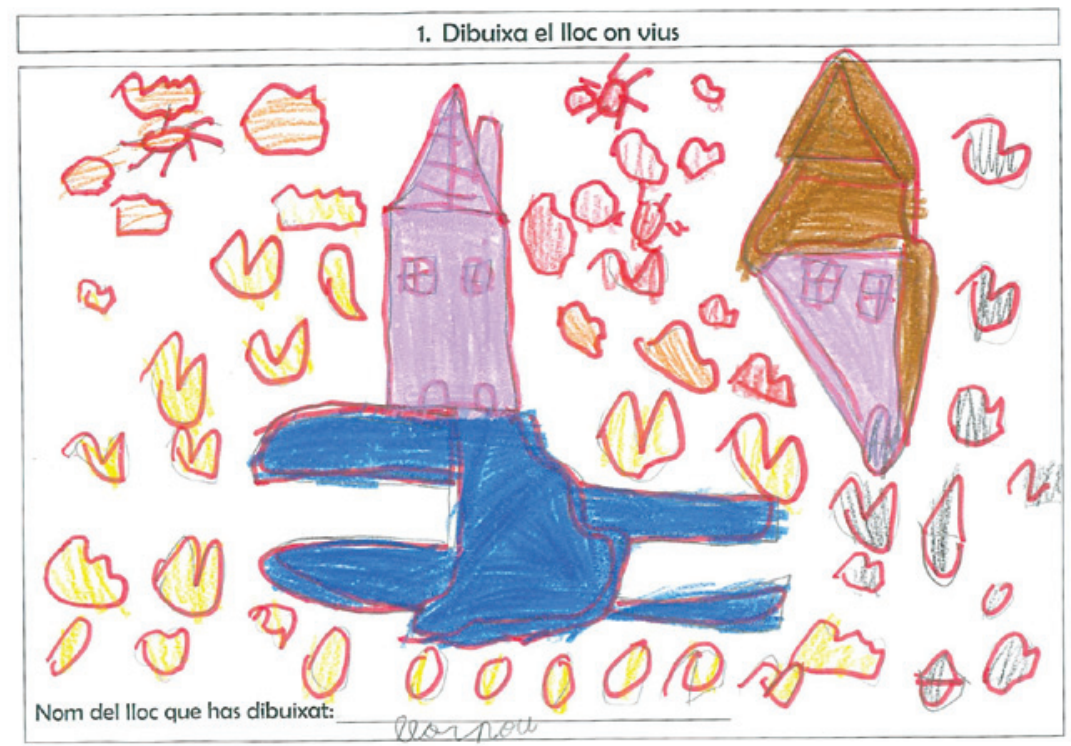

FIGURA N $^{\mathrm{o}} 1$. Representación escolar del espacio vivido en la etapa de Infantil. Fuente: Representación pictórica elaborada por el alumno 4

En segundo lugar, observaremos la Figura 2, en la que la alumna 2 ha representado un lugar muy, muy, pero que muy lejano. En su dibujo se aprecia Granada (Sierra Nevada) como lugar que percibe lejano, ya que ella lo ha conocido gracias a un viaje familiar y recuerda que no estaba cerca de dónde vive. Por tanto, la alumna representa un paisaje 
invernal con las nubes oscuras y la nieve en forma de muñecos o copos, además del hotel donde se hospedaron. Así y como se ha visto en el anterior dibujo, no se han representado elementos de la naturaleza ni seres vivos o personas. De este modo, observamos que para representar este espacio percibido, la alumna ha tenido que utilizar su experiencia subjetiva en un viaje que ha realizado y experimentado en primera persona.

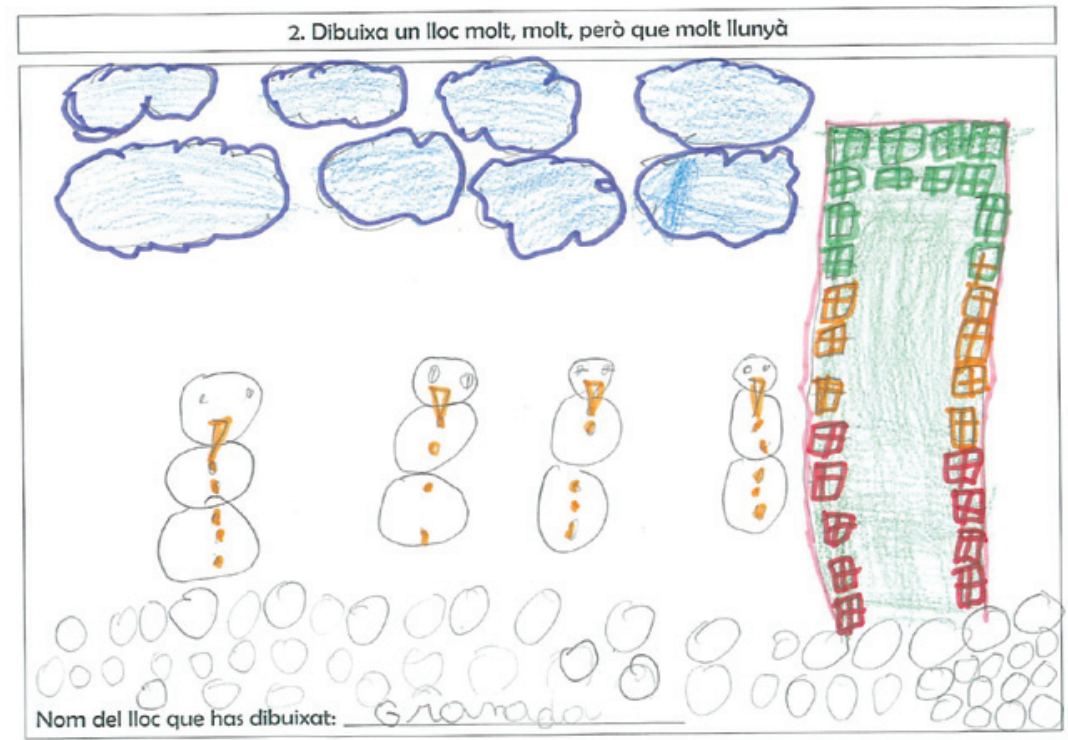

FIGURA N ${ }^{\circ} 2$. Representación escolar del espacio percibido en la etapa de Infantil. Fuente: Representación pictórica elaborada por la alumna 2

En tercer lugar, también encontramos cuestiones relacionadas con la comprensión de la complejidad espacial en las representaciones pictóricas analizadas, tal y como podemos observar en la Figura 3, en la que el alumno 4 representa como lugar muy, muy pero que muy lejano la ciudad de Londres, la cual visitó en un viaje familiar. Así pues, se observa que se trata de un dibujo muy interesante que nos hace abrir los ojos hacia la gran imaginación y percepción del alumnado de Educación Infantil, ya que el alumno considera que el mismo planeta Tierra (dibujado en el centro) es un espacio lejano, cosa que nos hace pensar que pueda estar influenciado por las vistas que tuvo desde el avión cuando visitó esta ciudad o por la imagen que ha visto constantemente en los dibujos, imágenes o televisión. Además, podemos observar también que representa muchas casas, las cuales recuerdan a los barrios londinenses por estar agrupadas por colores, ya que su recuerdo se centra en las dimensiones tan grandes de esta ciudad en comparación con su medio local y en la abundancia de casas y calles. Así, al igual 
que en las representaciones anteriores, no se han dibujado seres vivos, naturaleza o personas. Por tanto, de este modo, es necesario recalcar que este alumno goza de una avanzada concepción espacial, ya que en su dibujo se puede percibir claramente la existencia de una relación entre lo local y lo global, desde nuestro punto de vista.

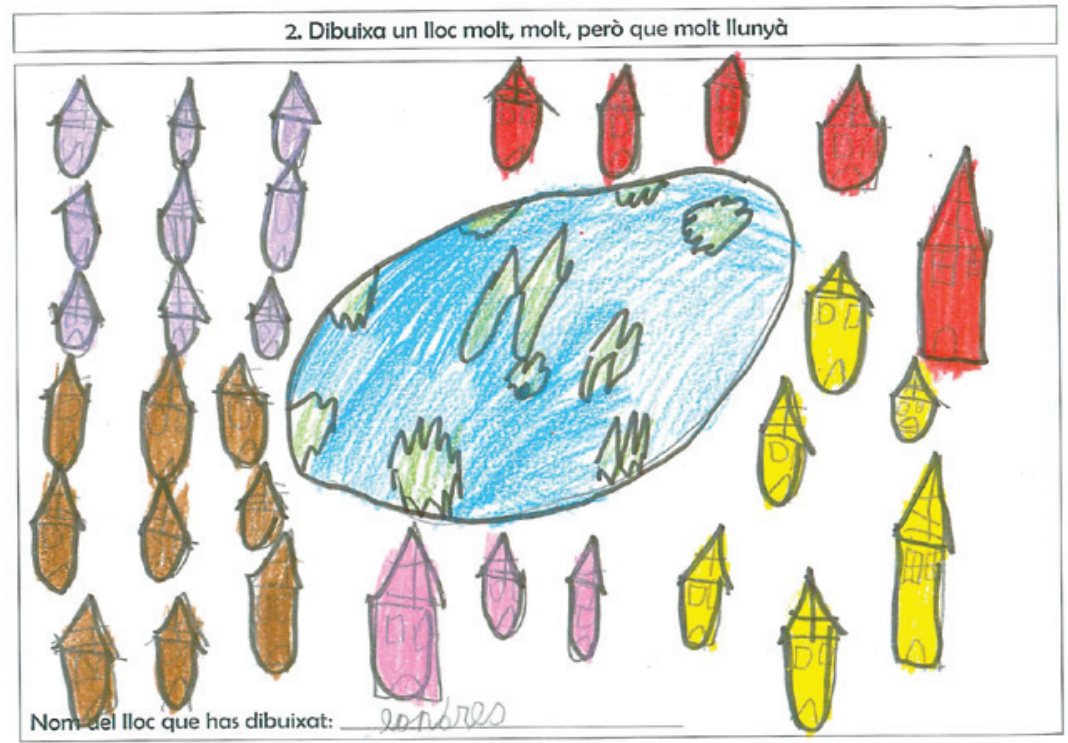

Figura $\mathrm{N}^{\mathrm{O}} 3$. Representación escolar donde se conecta lo local con lo global. Fuente: Representación pictórica elaborada por el alumno 4

Y, por último, si observamos la Figura 4, vemos que el alumno 6 se representa en un lugar muy, muy pero que muy lejano. En este caso se identifica una montaña muy alta que denomina Everest, donde se aprecia la influencia del cuento de la Fase de Intervención en el que se hacía referencia a la citada montaña. Se observa que el alumno se ha dibujado en la cima de la montaña junto a su familia, su madre y su padre, y además, al lado de la montaña ha dibujado también un parking, visto desde una perspectiva aérea, lleno de coches dónde identifica el suyo al pintarlo de negro. Por esta razón, observamos que esta representación aunque influenciada por el cuento, ha incorporado elementos que nos hacen pensar que también se puede basar en alguna montaña que el alumno ha visitado por la incorporación de su familia y de las vistas proporcionadas del parking que tuvo desde la cima. En esta representación vemos la gran capacidad de imaginación que tiene el alumnado de Educación Infantil para localizarse en lugares en los que nunca han estado y, sobre todo, la gran capacidad de concebir espacios tan lejanos como, en este caso, el Everest. 


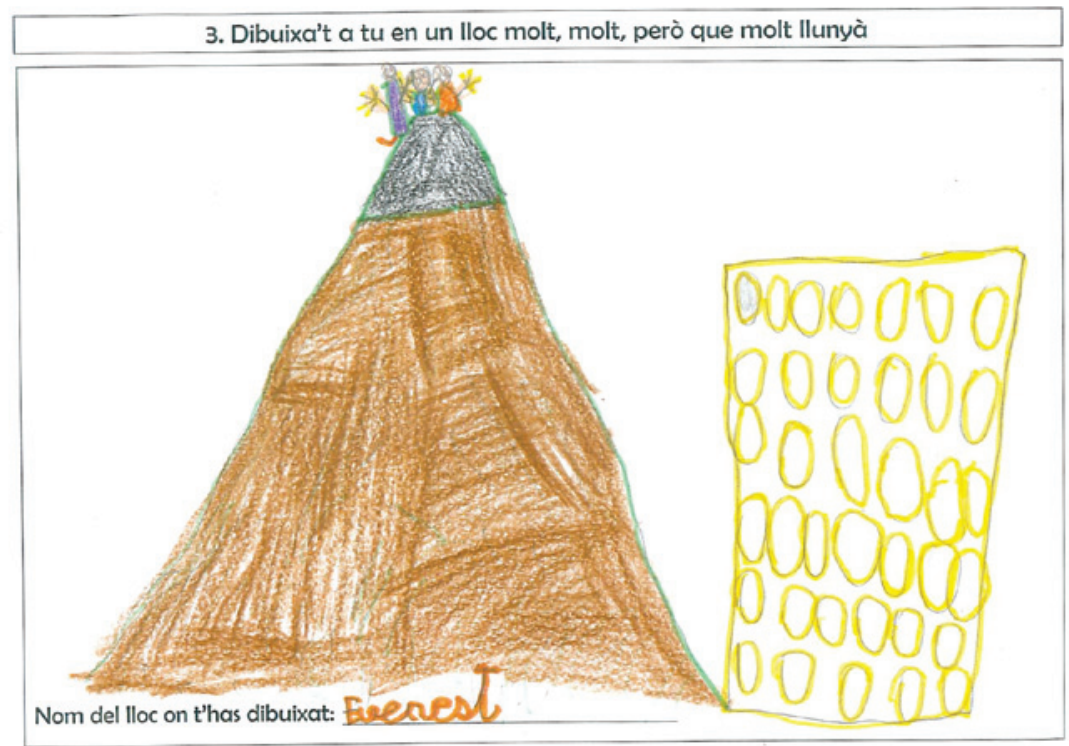

FigURA N ${ }^{\circ} 4$. Representación escolar del espacio concebido en la etapa de infantil. Fuente: Representación pictórica elaborada por el alumno 6

\subsection{Identificación en los dibujos de los conceptos relacionados con el pensamiento geográfico}

En la tabla 2 se muestra como los expertos/as han identificado en los dibujos los conceptos básicos que según Lana de Souza Cavalcanti (2017), definen el pensamiento geográfico: lugar, paisaje y territorio.

Según estos datos el alumnado de Educación Infantil representa en sus dibujos lugares, paisajes y territorios tanto en los entornos más próximos como en los más lejanos. Construye este pensamiento geográfico a partir de sus propias experiencias y de los recursos que tiene a su alcance para representar los lugares que vive y conoce, los que conoce y recuerda, así como los que desconoce y concibe a través de su imaginación. En el caso del paisaje, este ya no es solo un escenario natural que puede ser percibido, sino que aparece también en los lugares vividos y concebidos. Se muestran paisajes naturales pero también sociales, culturales e imaginarios. Por su parte el conocimiento de territorio conlleva intuir las invisibles relaciones que existen en el mismo, la compleja interacción entre lo natural y lo social, lo local y lo global o simplemente la relación entre los elementos que conforman el propio barrio. Esta construcción social del territorio aborda el espacio desde la propia percepción subjetiva a la que alude el Convenio Europeo del Paisaje, donde también se incluyen elementos relacionados con la identidad y las emociones. De ahí los territorios que han seleccionado porque les son significativos y se 


\begin{tabular}{|c|c|c|c|}
\hline \multirow{2}{*}{$\begin{array}{l}\text { REPRESENTACIONES } \\
\text { PICTÓRICAS }\end{array}$} & \multicolumn{3}{|c|}{$\begin{array}{l}\text { CONCEPTOS DEL PENSAMIENTO GEOGRÁFICO } \\
\text { (CAVALCANTI) }\end{array}$} \\
\hline & LUGARES & PAISAJES & TERRITORIOS \\
\hline Lugar dónde viven & $\begin{array}{l}\text { Aspectos naturales: } \\
\text { lago (1) } \\
\text { Aspectos } \\
\text { antrópicos: casas } \\
\text { (4), pueblo (1), } \\
\text { monumento o } \\
\text { castillo (1), pabellón } \\
\text { (1), piscina (1), } \\
\text { jardín o lugar de } \\
\text { juego (1) }\end{array}$ & $\begin{array}{c}\text { Aspectos naturales: } \\
\text { nubes (2), flores (1), sol } \\
\text { (1), animales (1), aves } \\
\text { (1), insectos (1) } \\
\text { Aspectos antrópicos: } \\
\text { casas (3), jardín, lago } \\
\text { o rincón de juego (2), } \\
\text { puente (1), pueblo: } \\
\text { zonas deportivas (1), } \\
\text { calle (1) }\end{array}$ & Llocnou (5) \\
\hline $\begin{array}{l}\text { Un lugar muy, muy, pero } \\
\text { que muy lejano }\end{array}$ & $\begin{array}{l}\text { Lugar concreto de } \\
\text { vacaciones con el } \\
\text { mar de fondo ( } 1)\end{array}$ & $\begin{array}{c}\text { Paisaje natural (3) } \\
\text { Paisaje antrópico (1) } \\
\text { Paisaje urbano (1) } \\
\text { Paisaje cultural (1) } \\
\text { Animales como } \\
\text { personajes animados (1) }\end{array}$ & África (1) \\
\hline $\begin{array}{l}\text { Alumno/a en un lugar } \\
\text { muy, muy, pero que muy } \\
\text { lejano }\end{array}$ & $\begin{array}{l}\text { Aspectos naturales: } \\
\text { animales (1) } \\
\text { Aspectos antrópicos: } \\
\text { sujeto en la cima } \\
\text { (2), sujetos (2), } \\
\text { viviendas (1), } \\
\text { pirámides (1), } \\
\text { persona (3), casa (2), } \\
\text { seres humanos (1) }\end{array}$ & $\begin{array}{c}\text { Aspectos naturales: } \\
\text { montañas (4), río (1), } \\
\text { sol (2), vegetación (2), } \\
\text { mar (1), naturaleza (1), } \\
\text { paisaje natural/artificial } \\
\text { (1), cima nevada (1), } \\
\text { cima montaña (1) } \\
\text { Aspectos antrópicos: } \\
\text { pirámides }(2)\end{array}$ & $\begin{array}{c}\text { India (2) } \\
\text { Everest (2) } \\
\text { Montañas (1) } \\
\text { Inglaterra (1) } \\
\text { Egipto (2) }\end{array}$ \\
\hline
\end{tabular}

TABLA N $^{\circ} 2$. Análisis de los aspectos de pensamiento geográfico evidenciados en los dibujos según los expertos/as. Fuente: elaboración propia / Nota: entre paréntesis se muestra la frecuencia de aparición de los elementos

identifican con los mismos, sobre todo en las dos primeras representaciones pictóricas. Respecto a la representación de ellos/as en "un lugar muy, muy, pero que muy lejano" cabe destacar la aparición de personas y en concreto de las familias para sentirse seguros/ as en esos lugares desconocidos. 


\subsection{Categorización de los espacios representados (vivido, percibido y concebido)}

El Juicio de Expertos nos ha facilitado una categorización de los espacios representados por el alumnado de Educación Infantil. Para ello les hemos pedido que en cada uno de los dibujos puedan diferenciar los conceptos básicos del pensamiento geográfico (lugar, paisaje y territorio) y que a su vez categoricen estos espacios en vividos, percibidos y concebidos, según la clasificación de Hannoun (1977). En la tabla 3 se puede observar que en principio hay una serie de dibujos que los expertos/as no pueden categorizar sin saber la explicación del alumnado de Educación Infantil. En el caso de la categorización de los espacios vividos y percibidos, existe una interrelación/interacción entre el lugar vivido y el paisaje que no

\begin{tabular}{|c|c|c|c|c|c|}
\hline \multirow{2}{*}{$\begin{array}{c}\text { LUGARES } \\
\text { REPRESENTADOS }\end{array}$} & \multirow{2}{*}{$\begin{array}{l}\text { RECURSOS } \\
\text { DIDÁCTICOS } \\
\text { UTILIZADOS }\end{array}$} & \multicolumn{4}{|c|}{$\begin{array}{c}\text { CATEGORIZACIÓN DE LOS ESPACIOS SIGUIENDO } \\
\text { LA CLASIFICACIÓN DE HANNOUN (1977) } \\
\text { Juicio de Expertos }\end{array}$} \\
\hline & & \begin{tabular}{|c|} 
ESPACIO \\
VIVIDO \\
\end{tabular} & $\begin{array}{c}\text { ESPACIO } \\
\text { PERCIBIDO }\end{array}$ & $\begin{array}{c}\text { ESPACIO } \\
\text { CONCEBIDO } \\
\end{array}$ & $\begin{array}{c}\text { No sabe/ No } \\
\text { contesta }\end{array}$ \\
\hline Lugar dónde viven & $\begin{array}{c}\text { Experiencias } \\
\text { locales }\end{array}$ & $\begin{array}{c}\text { Paisaje } \\
\text { (dibujos } 4 \\
\text { y } 16) \\
\text { Lugar } \\
\text { (dibujos } 7, \\
10 \text { y 13) }\end{array}$ & $\begin{array}{c}\text { Paisaje } \\
\text { (dibujos } 7,10 \\
\text { y 13) } \\
\text { Lugar (dibujos } \\
4 \text { y 16) }\end{array}$ & $\begin{array}{l}\text { Territorio } \\
\text { (dibujos } 4,7 \text {, } \\
10,13 \text { y } 16)\end{array}$ & \\
\hline $\begin{array}{l}\text { Un lugar muy, muy, } \\
\text { pero que muy lejano }\end{array}$ & $\begin{array}{c}\text { Viajes } \\
\text { Imaginación } \\
\text { Proyecto Egipto } \\
\text { Medios } \\
\text { comunicación } \\
\text { Experiencias } \\
\text { directas }\end{array}$ & $\begin{array}{l}\text { Lugar } \\
\text { (dibujo } \\
19)\end{array}$ & $\begin{array}{l}\text { Paisaje } \\
\text { (dibujos } 5,8 \\
14 \text { y } 17)\end{array}$ & $\begin{array}{c}\text { Territorio } \\
\text { (dibujos } 5 \text { y } 11)\end{array}$ & $\begin{array}{c}\text { Lugar } \\
\text { (dibujos 5, } 8, \\
11,14 \text { y 17) } \\
\text { Paisaje } \\
\text { (dibujo 19) } \\
\text { Territorio } \\
\text { (dibujos } \\
8,11,14,17 \\
\text { y 19) }\end{array}$ \\
\hline $\begin{array}{l}\text { Alumnola en un lugar } \\
\text { muy, muy, pero que } \\
\text { muy lejano }\end{array}$ & $\begin{array}{c}\text { Cuento } \\
\text { Intervención } \\
\text { Proyecto Egipto } \\
\text { Imaginación } \\
\text { Familia } \\
\text { Experiencias } \\
\text { directas } \\
\end{array}$ & 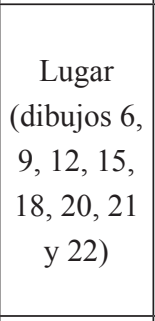 & $\begin{array}{c}\text { Paisaje } \\
\text { (dibujos 6, 9, } \\
\text { 12, 15, 18, } 20 \\
\text { y 21) } \\
\text { Territorio } \\
\text { (dibujo 22) }\end{array}$ & $\begin{array}{c}\text { Paisaje (dibujo } \\
\text { 22) } \\
\text { Territorio } \\
\text { (dibujos } 6,912, \\
15,18 \text { y } 21 \text { ) }\end{array}$ & \\
\hline TOTAL & & 14 & 17 & 15 & 11 \\
\hline
\end{tabular}

TABLA 3. Análisis del espacio vivido, percibido y concebido en los dibujos según los expertos/as. Fuente: elaboración propia. 
responde a los conceptos de proximidad/cercanía propio de la construcción de un espacio geométrico siguiendo los postulados de Hannoun sino que más bien tiene que ver con una construcción social del espacio (Rivero y Gil, 2011), donde tiene cabida la visión subjetiva de las personas. Respecto al espacio concebido Hannoun hace referencia a la capacidad de abstracción lógico-matemática en el ámbito de la psicología evolutiva para comprender el espacio geométrico. Mientras que los expertos/as, en este estudio de casos lo relacionan con el territorio, desde una construcción social, en el que tiene cabida no solo el espacio absoluto tal y como plantea el constructivismo sino también el espacio subjetivo donde se evidencian relaciones de identidad y emociones que ayuden al alumnado de Educación Infantil a la construcción de un "espacio común" (Rivero y Gil, 2011:41) coherente desde un punto de vista sostenible y solidario.

Respecto a los recursos didácticos utilizados por el alumnado para realizar sus representaciones pictóricas, observamos que los dibujos del lugar dónde viven, están influenciados mayoritariamente por la cotidianidad de su casa y su pueblo o calle; en cuanto a los dibujos del lugar muy lejano, están influenciados principalmente por el Proyecto de Egipto del aula y las experiencias en viajes o la televisión; y, por último, los dibujos de los alumnos en lugares muy lejanos, están influenciados mayoritariamente también por el Proyecto de Egipto, por la creatividad o imaginación propia del alumnado y por el cuento de la Fase de Intervención. Por lo tanto, es necesario resaltar que abrir el mundo a los ojos de los más pequeños/ a través de historias, trabajos, imágenes o videos, ayuda al desarrollo de capacidades que posibiliten el pensamiento geográfico en estos primeros años de la infancia donde la imaginación y la fantasía, tal y como evidenciaba Egan (2007) juegan un papel muy importante para poder concebir y construir un espacio social en el que ellos/as puedan participar de una ciudadanía de pleno derecho.

\section{CONCLUSIONES}

Esta investigación adolece de las limitaciones propias de un estudio de casos pero a su vez muestra una serie de indicios que evidencian la verificación de la hipótesis al comprobar que el alumnado de $2^{\circ}$ ciclo de Educación Infantil es capaz de desarrollar el pensamiento geográfico. Las consideraciones más significativas que se desprenden de este trabajo pueden resumirse de la siguiente manera:

- Según este estudio de casos, la evolución de la compresión espacial en la etapa de Educación Infantil responde a la construcción social del espacio que desde sus propias experiencias elaboran los niños/as, utilizando la imaginación para comprender y concebir los espacios más complejos. Distinguen claramente lo próximo de lo lejano en función de lo que han vivido y percibido. Además son capaces de representarse a ellos/as mismos/as en un espacio donde no han estado jamás. 
- En sus representaciones pictóricas son capaces de dibujar el lugar, el paisaje y el territorio, conceptos básicos para el desarrollo del pensamiento geográfico, según Lana de Souza Cavalcanti (2017), tanto en los entornos más próximos como en los más lejanos. Construyen este pensamiento geográfico a partir de sus propias experiencias y de los recursos que tienen a su alcance para representar los lugares que conocen y recuerdan, así como los que desconocen y conciben mediante su imaginación.

- A través de sus propias experiencias y la imaginación son capaces de representar el espacio vivido, percibido y concebido como una construcción social, donde elementos como la identidad, las emociones, relaciones entre lo local y lo global y la territorialidad, que requieren una gran capacidad de abstracción han quedado reflejados en sus dibujos.

- La utilización de recursos didácticos como proyectos de aula, cuentos y actividades que fomenten su imaginación les ayudan a concebir el espacio y a potenciar su espacio subjetivo.

- La implementación en el aula de estos recursos educativos con modelos didácticosevaluativos que permitan la participación del alumnado de Educación Infantil en la construcción del espacio geográfico favorece una correcta interpretación de lo que saben los niños/as para que los/las docentes les puedan ayudar a conectar estos espacios subjetivos con los absolutos.

\section{BIBLIOGRAFÍA}

Abric, J. C., 1993. Prácticas sociales y representaciones. París: Presses Universitaires de Francia.

Andrade, E. y Moreira, K.F., 2017. "Representações sociais de pobreza construídas pelos cursistas da Especialização Educação, Pobreza e Desigualdade Social realizada no Rio Grande do Norte: primeiros resultados". Educar em Revista, 2, pp. 35-54.

Barraza, L., 1999. "Children's drawing about the environment". Environmental Education Research. Bath. Vol. 5 (1), pp. 49-67.

Boira, J.V., 2005. "La percepción del paisaje como instrumento imprescindible para el ingeniero paisajista" en J.F. Ballester Olmos. Proyectos de Regeneración del Paisaje. Valencia: Escuela Técnica Superior del Medio Rural y Enologia (UPV), pp. 53-64.

Boira, J.V., Reques, P. y Souto, X.M., 1994. Espacio subjetivo y geografía: orientación teórica y praxis didáctica. València: Nau Llibres.

Capel, H., 1981. Filosofía y ciencia en la Geografía contemporánea. Barcelona: Barcenova.

Cavalcanti, L., 2017. "El análisis de la espacialidad y la comprensión del mundo: llave para la relevancia de la geografía escolar", en R. Sebastià y E.M ${ }^{a}$ Tonda, coord. Enseñanza y aprendizaje de la geografía para el siglo XXI. Alicante: Universidad de Alicante, pp. 97-116. 
Comes, P., 2002. "La interpretación de las imágenes espaciales" en La Geografía y la Historia, elementos del medio. Madrid: Ministerio de Educación, Cultura y Deporte, pp. 209-244.

Consejo de Europa, 2000. Convenio Europeo del Paisaje. Florencia: Ministerio de Asuntos Exteriores.Disponible en cultura/patrimonio/Convenio_europeo_paisaje. pdf [Último acceso 13 febrero 2018]

Denzin, N. K., 1970. Sociological Methods: a Source Book. Chicago: Aldine Publishing Company.

Donet, E., 2016. Pensar històricament en Educació Infantil. Un estudi de cas. Trabajo Final de Máster inédito. Valencia: Universidad de Valencia.

Egan, K., 2007. Fantasía e imaginación: su poder en la enseñanza. Una alternativa a la enseñanza y aprendizaje en Educación Infantil y Primaria. Madrid: Morata y Ministerio de Educación y Ciencia.

Gardner, H., 2005. Inteligencias múltiples: la teoría en la práctica. Barcelona: Paidós Ibérica.

Goodey, B., 1973. Perception of the Environment: an introduction to the literature. Birmingham: Occasional Paper $n^{\circ} 1,17$, University of Birmingham.

Gould, P., 2000. "Pensar como un geógrafo, una exploración en la geografía moderna". Scripta Nova, Revista Electrónica de Geografía y Ciencias Sociales, 78. Barcelona: Universidad de Barcelona. Disponible en http://www.ub.edu/geocrit/ sn-78.htm [Último acceso 11 febrero 2018].

Hannoun, H., 1977. El niño conquista el medio. Buenos Aires: Kapelusz.

Hernández, F.X., 2002. Didáctica de las Ciencias Sociales, Geografía e Historia. Barcelona: Graó.

Luis, A. y Urteaga, L., 1982. "Estudio del medio y heitmakunde en la geografía escolar". Geocrítica, Cuadernos Críticos de Geografía Humana, 38. Disponible en http:// www.ub.edu/geocrit/geo38.htm [Último acceso 15 enero 2018].

Lynch, K., 1960. The Image of City. Cambridge: The Technology Press \& Harvard University Press.

Mateos, J., 2001. “Genealogía del código pedagógico del entorno”. Aula, 13, pp. 19-35.

Moscovici, S., 1978. La psychanalyse, son image, son public. Paris: PUF.

Nadal, I., 1999. La concepción del espacio próximo y lejano. Justificación de una propuesta alternativa para el estudio del medio en Primaria. Tesis Doctoral inédita. Las Palmas de Gran Canaria: Universidad de Las Palmas de Gran Canaria.

Ortiz, A., Prats, M. y Baylina, M., 2011. Métodos visuales y geografías de la infancia: dibujando el entorno cotidiano. Scripta Nova, Revista Electrónica de Geografía y 
Ciencias Sociales, vol. XVI, 400. Barcelona: Universitat de Barcelona. Disponible en: http://www.ub.edu/geocrit/sn/sn-400.htm [Último acceso 22 enero 2018].

Pérez, P. y Souto González, X. M., 1989. "Bases para el conocimiento del medio en la enseñanza Primaria: una alternativa desde la Comunidad Valenciana". Ponencia presentada en Jornadas de Didáctica de Ciencias Sociales. Valladolid: ICE.

Piaget, J. e Inhelder, B., 1947. La représentatition de l'espace chez l'infant. Paris: PUF. Pieper, C. (2000). Fent un tomb pel món, Caterina i l'Ós. Barcelona: Kalandraka Editora. Piñeiro, M. R. 1983. "El entorno y su valor pedagógico". Aula Abierta, 38, pp. 79-87.

Rivero, Ma . P. y Gil, J., 2011. "Pensar y expresar el espacio en el aula de Infantil”, en $\mathrm{M}^{\mathrm{a}} \mathrm{P}$. Rivero, coord. Didáctica de las Ciencias Sociales para Educación Infantil. Zaragoza: Mira editores, pp. 31-47.

Souto, X.M., 2011. "La construcción del conocimiento escolar en la sociedad de comunicaciones. Una propuesta del proyecto Gea-Clío". Investigación en la Escuela, 75, pp. 7-19.

Souto, X.M. y García Monteagudo, D., 2016. "La geografía escolar ante el espejo de su representación social”. Didáctica Geográfica, 17, pp. 177-201.

Tonda, E.Mª,, 2009. "La geografía en el grado de maestro de educación infantil en España” en VVAA. A Inteligência Geográfica na Educação do Século XXI. Lisboa: Associaçao de Professores de Geografia; Instituto de Geografía e Ordenamento do Territorio da Universidade de Lisboa; Grupo de Didáctica de la Geografía de la Asociación de Geógrafos Españoles, pp. 1-23.

Thurstone, L., 1938. Primary mental abilities. Chicago: University of Chicago Press.

Trepat, C. y Comes, P., 1998. El tiempo y el espacio en la didáctica de las ciencias sociales. Barcelona: Graó.

Tonucci, F., 1993. "Hacia una propuesta de educación ambiental coherente". Aula de Innovación Educativa, 12, pp. 82-85. Barcelona: Graó.

Tonucci, F. 1995. "El niño y la ciencia" en Con ojos de Maestro, Gladys Kochen (trad.), Buenos Aires: Troquel (Serie Flacso acción), pp. 85-107. Disponible en <http:// www.zona-bajio.com/EyCM_anexo1.pdf > [Último acceso 9 noviembre 2017].

Urkidi, P., 1994. "La geografía, fundamento epistemológico y aplicación didáctica". Lurralde: investigación y espacio, 17, pp.153-191 Disponible en: http://www. ingeba.org/lurralde/lurranet/lur17/17urkidi.htm/17urkidi.htm [Último acceso 15 enero 2018].

Vigotsky, L.S., 1962. Thought and Languaje. Cambridge: The MLT Press. 